\title{
Pericholangitis with Ulcerative Colitis Following Autoimmune Hepatitis over 12 Years
}

\author{
Seiko Chujo, Choitsu Sakamoto, Shigekazu Ohno, Shinsuke Morimoto, Masaki Miyamoto, \\ Hiroyuki Okano, Sumiharu Morita, Susumu Saeki, Munetada Oimomi, Takahiro Fujimori*, \\ Sakan MaEdA* and Masato Kasuga
}

\begin{abstract}
A 35-year-old woman was diagnosed as autoimmune hepatitis 12 years ago by abnormal findings of liver tests including lupus erythematosus (LE) cell phenomenon and liver biopsy. She was admitted in May 1990 with a history of lower gastrointestinal bleeding. Colonoscopy with biopsy and barium enema revealed chronic ulcerative colitis along the entire colon. Since liver tests did not respond well to prednisolone treatment, liver biopsy was again performed and it revealed periductal inflammation with small duct proliferation, a finding compatible with pericholangitis. We herein report this patient who was initially diagnosed as autoimmune hepatitis and thereafter found to be pericholangitis associated with ulcerative colitis.

(Internal Medicine 31: 1228-1232, 1992)
\end{abstract}

Key words: liver biopsy, colonoscopy, ERCP

\section{Introduction}

Chronic active liver disease with lupus erythematosus (L.E.) cell phenomenon has been considered to be in a category referred to as autoimmune hepatitis (1). Other autoimmune diseases such as ulcerative colitis $(2,3)$, may be associated with the disease during the clinical course. Although the disease is almost always responsive to corticosteroids, many patients eventually relapse years later as autoimmune hepatitis. However, it is not well known that pericholangitis, the characteristic of which is peri-ductal inflammation in the portal fields, may occur after a longterm clinical course of autoimmune hepatitis.

We recently treated a patient who was initially diagnosed as autoimmune hepatitis and thereafter found to be pericholangitis by liver biopsy and clinical features. Furthermore, the patient was found to have accompanying chronic ulcerative colitis. We present this case since it is interesting that autoimmune hepatitis was clinically and histologically given place to pericholangitis which seems to be associated with ulcerative colitis $(4,5)$.

\section{Case Report}

A 35-year-old Japanese female was admitted to Kobe
University Hospital in May 1990 with a 5-day previous history of massive bleeding from the lower gastrointestinal tract accompanied by hypovolemic shock.

In February 1979, she had been admitted to Kobe University Hospital because of low grade fever and jaundice. At that time, she was diagnosed as autoimmune hepatitis on the basis of liver tests with liver biopsy. The serum alkaline phosphatase activity $8 \mathrm{U}$ (Bessy Lowry method, normal 1.2-2.9); sGOT $820 \mathrm{KU}$ (normal 8-40), sGPT $457 \mathrm{KU}$ (normal 5-35), total bilirubin $2.0 \mathrm{mg} / \mathrm{dl}$, total protein $10.4 \mathrm{~g} / \mathrm{dl}$, albumin $3.4 \mathrm{~g} / \mathrm{dl}$ and a diffuse hypergammaglobulinemia with $\mathrm{IgG}$ of $5628 \mathrm{mg} / \mathrm{dl}, \operatorname{IgA}$ of $447 \mathrm{mg} / \mathrm{dl}$ and $\mathrm{IgM}$ of $172 \mathrm{mg} / \mathrm{dl}$. Anti-nuclear antibody, anti-DNA antibody and anti-smooth muscle antibody were all positive. Although LE cell phenomenon was absent at that time, one of four LE preparations became positive later. Specimens of a liver biopsy performed showed infiltration into the hepatic lobules of inflammatory cells mainly constituting of plasma cells, suggesting the existence of chronic active hepatitis (Fig. 1). Hepatitis B serologies were absent. Therefcre, she was diagnosed as autoimmune hepatitis and treated with prednisolone $30 \mathrm{mg}$ daily for 4 weeks. She responded well to the treatment and she was discharged on prednisolone $10 \mathrm{mg}$ daily on May 1981 (Fig. 2). Thereafter, she continued to undergo medical follow-up.

From the Sccond Department of Internal Medicine, and the *the Second Department of Pathology, Kobe University School of Mcdicine, Kobe Received for Publication March 18, 1992; Accepted for publication August 25, 1992

Reprint requests should be addressed to Dr. Choitsu Sakamoto, the Second Department of Internal Medicine, Kobe University School of Medicine, Kusunoki-cho, Chuo-ku, Kobe 650, Japan 

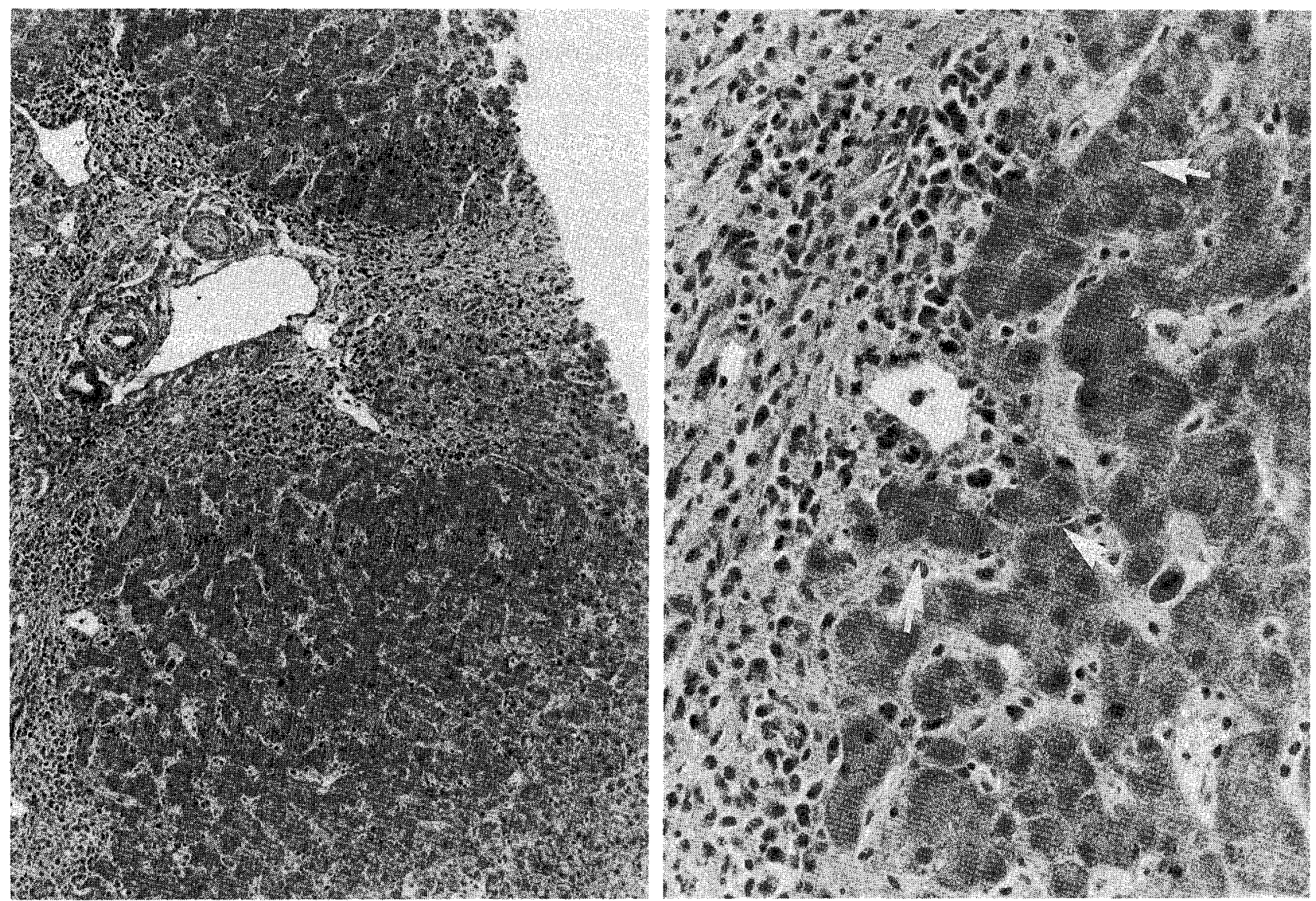

Fig. 1. Hematoxylin-eosin staining of the liver biopsy specimen obtained on initial admission. Left panel $(10 \times 2.5)$ : Expansion of inflammatory cells such as plasma cells and lymphocytes into hepatic lobule is the prominent feature. Right panel $(20 \times 4)$ : Arrows indicate destruction of hepatocytes located around the lobule.

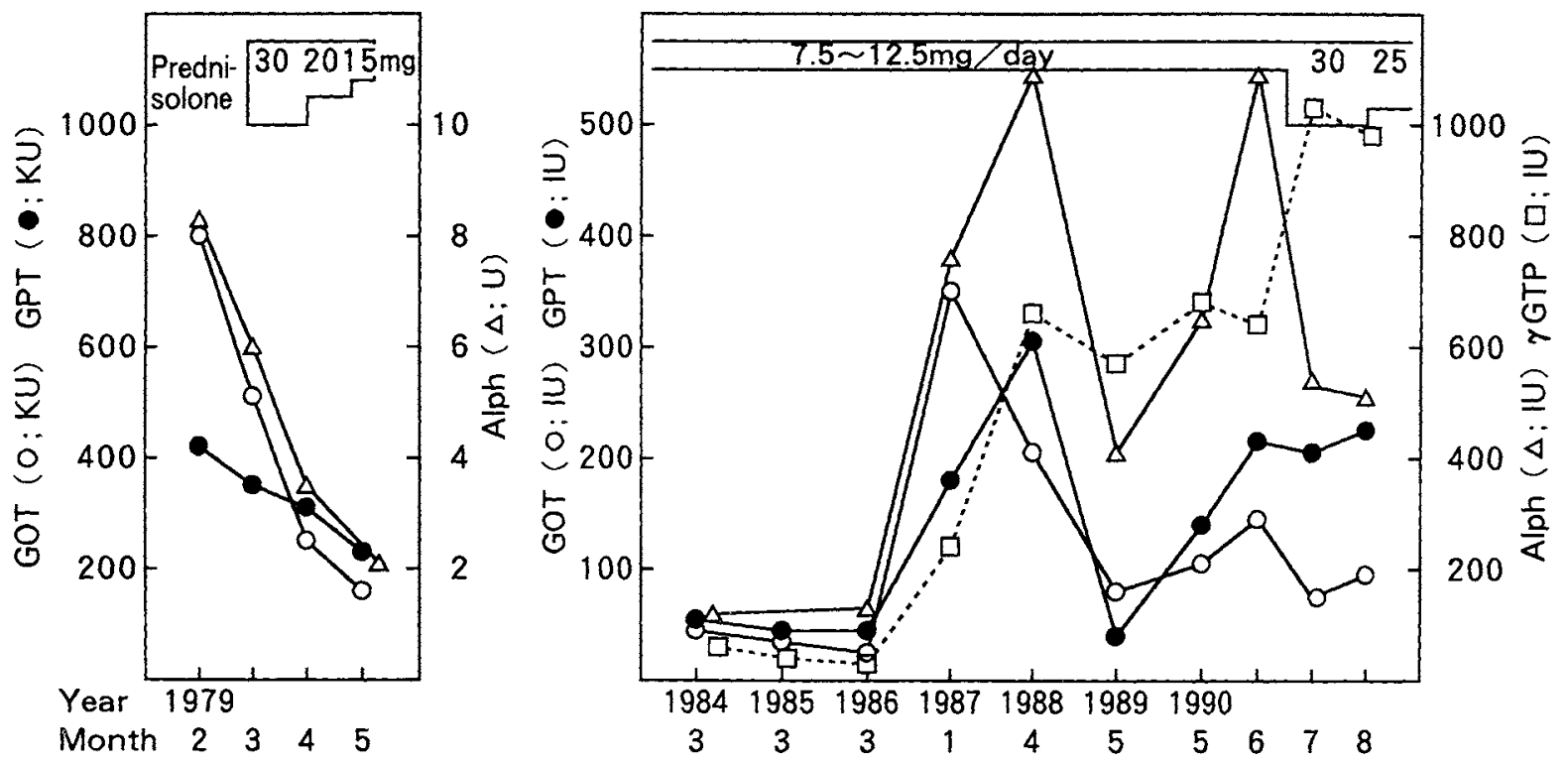

Fig. 2. Clinical time course of liver tests in response to prednisolone treatment. 
The patient occasionally experienced recurrent episodes of diarrhea during the 6 subsequent years. Since 1987, the activities of serum alkaline phosphatase and $\gamma$-glutamyl transpeptidase gradually became elevated even though the patient continued to take $7.5 \mathrm{mg}-12.5 \mathrm{mg}$ of prednisolone daily (Fig. 2). Six days prior to admission in May 1990, there were no clinical symptoms such as diarrhea, abdominal pain or fever.

On admission, she had no guarding of abdominal muscles and tenderness, but an elastic firm non-tender and non-nodular liver was palpable with a span of $1.5 \mathrm{~cm}$ in the mid clavicular line. Rectal examination revealed no mass, but the stool showed had a trace of blood. Initial laboratory data included an ESR of $46 \mathrm{~mm} / \mathrm{h}$, hemoglobin $9.7 \mathrm{~g} / \mathrm{dl}$, a white blood cell count $9,900 / \mathrm{mm}^{3}$, and a platelet count $323,000 / \mathrm{mm}^{3}$. The serum alkaline phosphatase activity was $664 \mathrm{IU} / \mathrm{l}$; total bilirubin $0.7 \mathrm{mg} / \mathrm{dl}$, sGOT $103 \mathrm{IU} / 1$, sGPT $139 \mathrm{IU} / 1$, $\gamma$-glutamyl transpeptidase $671 \mathrm{IU} / \mathrm{l}$, total protein $6.0 \mathrm{~g} / \mathrm{dl}$ and albumin $3.3 \mathrm{~g} / \mathrm{dl}$. IgG was $1643 \mathrm{mg} / \mathrm{dl} ; \mathrm{IgA} 260 \mathrm{mg} / \mathrm{dl}$ and $\mathrm{IgM} 166 \mathrm{mg} / \mathrm{dl}$. Anti-nuclear antibody was still positive, but anti-smooth muscle and anti-DNA antibodies were absent this time. Anti-mitochondrial antibody was also absent. Hepatitis B and Hepatitis $C$ virus serologies were all absent. Barium enema showed a slight shortening of the bowel with inflammatory polyps. Colonoscopy to ceacum revealed slightly dry mucosa with inflammatory polyps and indistinct vascular pattern of the mucosa along the entire colon and rectosigmoid, although it did not reveal the location of the bleeding (Fig. 3). Pathological examination of a colonic biopsy revealed distorted crypt architectures and crypt atrophy with inflammatory cell infiltration, a finding consistent with chronic ulcerative colitis (data not shown). On the 30th hospital day, prednisolone was increased to $30 \mathrm{mg}$ daily for the treatment of liver dysfunction and chronic ulcerative colitis. However, sGOT and sGPT did not return to normal and $\gamma$-glutamyl transpeptidase activity increased from $669 \mathrm{IU} / 1$ to $1,092 \mathrm{IU} / 1$ in the course of next 4 weeks (Fig. 2). To clarify unresponsiveness of liver tests to prednisolone and compare pathological findings of the liver with those obtained previously, a liver biopsy was performed again and revealed a finding compatible with pericholangitis. There was no expansion of inflammatory cells into the hepatic lobule and no destruction of the hepatocytes located around the lobule (Fig. 4). Endoscopic retrograde cholangiography showed that the common bile duct was possibly partially sclerotic, although primary sclerosing cholangitis could not be diagnosed by these findings (Fig. 5). Since the patient had no major systemic complaints, prednisolone was decreased to $15 \mathrm{mg}$ per day 4 months after admission. Although the patient continued to take $12.5 \mathrm{mg} \sim 15 \mathrm{mg}$ of prednisolone per day thereafter, liver dysfunction (alph 477 IU, ₹GTP 262 IU, sGOT 58 IU, sGPT $100 \mathrm{IU}$ ) is still present in January 1992.
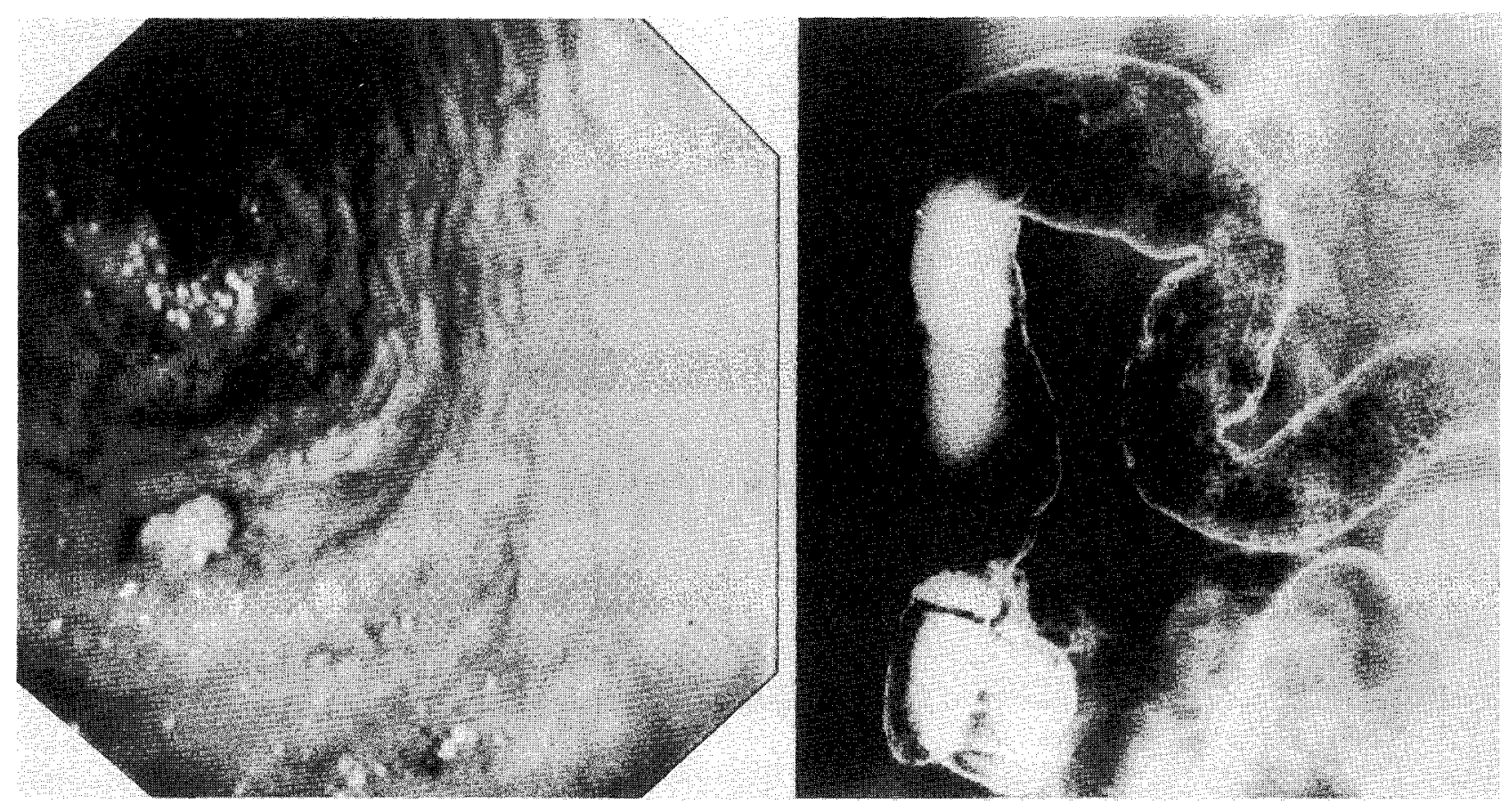

Fig. 3. Left pancl: Colonoscopic photograph showing a representativc lesion of dry and granular mucosa with an inflammatory polyp and indistinct vascular pattern in the transverse colon. Right pancl: Barium encma showing inflammatory polyps and a decreased mucosal pattern in the transverse colon. 

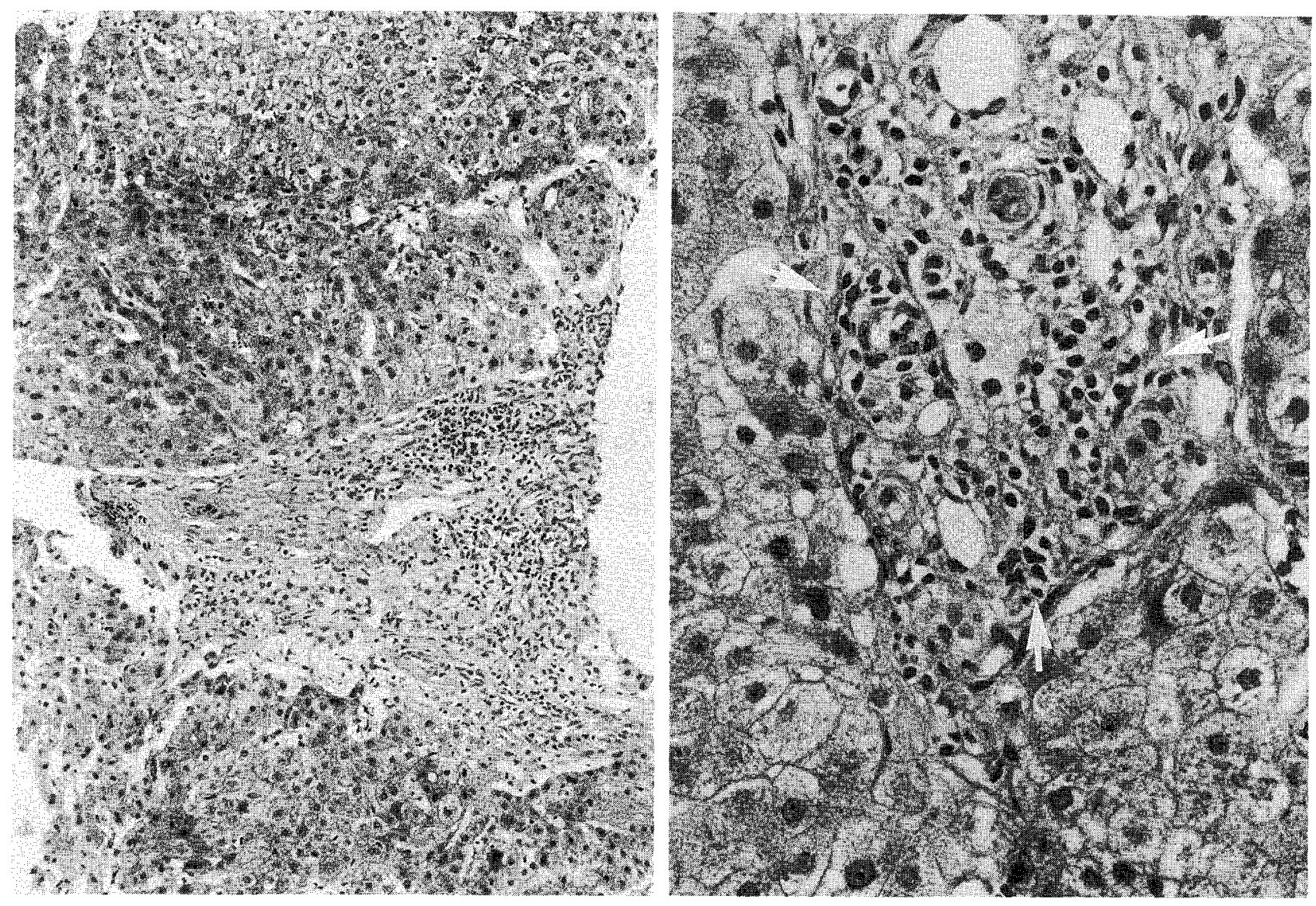

Fig. 4. Hematoxylin-eosin staining of the liver biopsy specimen obtained on second admission. Left panel $(10 \times 2.5)$ : portal field shows mild pericholangitis with lymphocyte infiltration. Right panel $(20 \times 4)$ : High power view of the portal field. Arrows clearly indicate remarkable infiltration of lymphocytes around small bile ducts. There is no destruction of hepatocytes located around the hepatic lobule.

\section{Discussion}

We presented a patient whose clinical features of pericholangitis associated with ulcerative colitis were complicated by her past history of autoimmune hepatitis. The patient was initially diagnosed by liver tests with liver biopsy as autoimmune hepatitis. The diagnostic criteria including an abnormally high concentration of IgG had been clinically and histologically satisfied at that time. Of interest is the observation that the symptoms and signs of autoimmune hepatitis gradually changed during the clinical course of 12 years. As far as we know, there is no report available which discusses alteration from autoimmune hepatitis to pericholangitis clinically and histologically. This time, abnormally high activities of serum alkaline phosphatase and $\gamma$-glutamyl transpeptidase were found. The recovery of such abnormalities of liver function tests was very poor in response to prednisolone treatment as compared with that of an initial treatment. The present case do not seem to be primary biliary cirrhosis, due to the fact that anti-mitochondrial antibody was absent and liver biopsy specimens did not show non-suppurative destructive cholangitis. On the other hand, the liver biopsy specimen showing periductal lymphocyte infiltration suggests that this patient may be in the first stage of primary sclerosing cholangitis. Recently, it has been suggested that the histological features of pericholangitis may be identical to the liver histology of patients with primary sclerosing cholangitis (6). 65 to $75 \%$ of the primary sclerosing cholangitis may occur in the patients with inflammatory bowel diseases, especially chronic ulcerative colitis (7). Furthermore, occurrence of primary sclerosing cholangitis or pericholangitis may be related to autoimmune mechanisms. Bodenheimer et al (8) have described elevated circulating immune complexes in patients with primary sclerosing cholangitis. Anti-smooth muscle and anti-nuclear antibodies have also been described in primary sclerosing cholangitis (9). Therefore, the present case raises the possibility that pericholangitis may be caused by autoimmune mechanisms after a long-term clinical course of autoimmune hepatitis.

It has been well known that liver disease is a common complication of chronic inflammatory bowel disease, especially chronic ulcerative colitis (10). Perrett et al (11) have shown that 45 patients $(15 \%)$ had an abnor- 


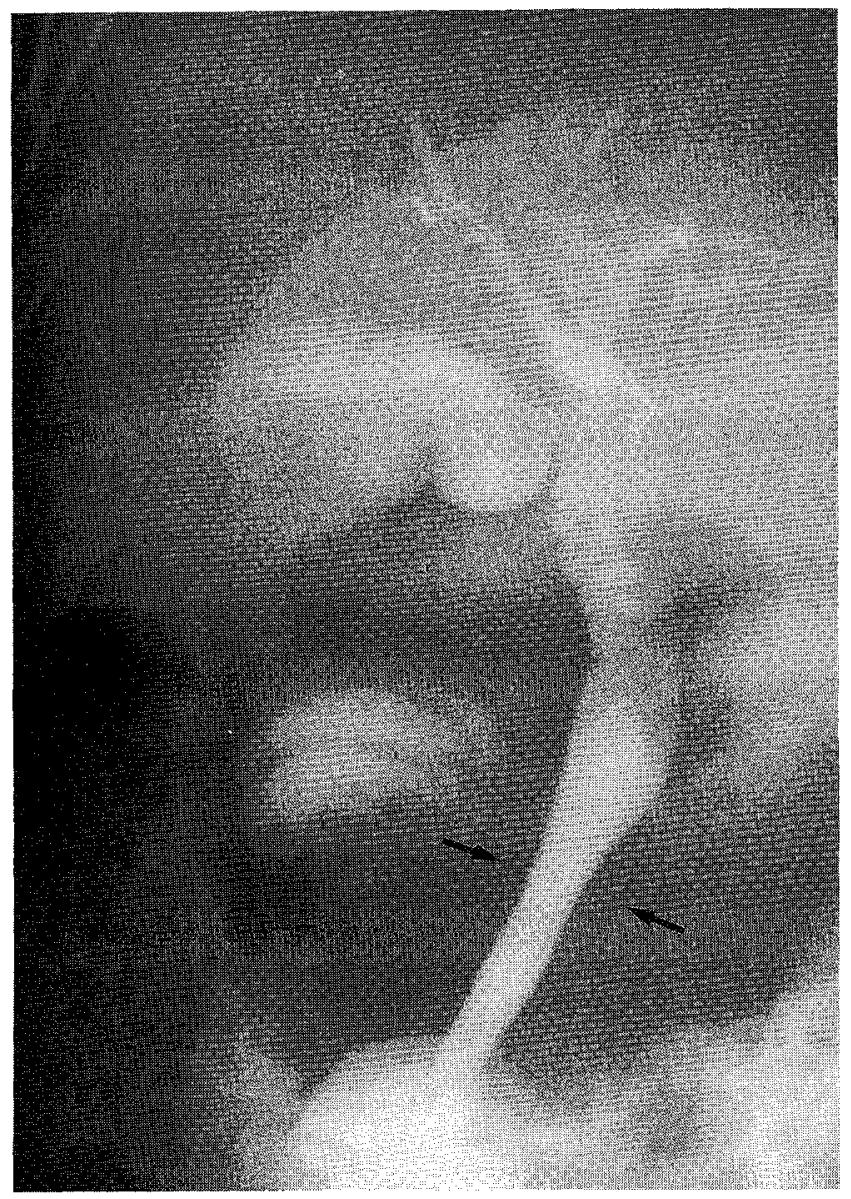

Fig. 5. Endoscopic retrograde cholangiography. Arrows indicate the portion which seems to be partially sclerotic in the common bile duct.

mality of one or more of basic liver tests among 300 patients with chronic ulcerative colitis. They revealed that pericholangitis occurred in 15 patients $(5 \%)$, although fatty change was the most common liver dysfunction occurring in 19 patients (6.3\%). Dordal et al (12) have reported that pericholangitis was diagnosed by liver biopsy in 33 of 720 patients with chronic inflammatory bowel diseases. The term pericholangitis was originally used to describe a morphologic feature of the syndrome of primary biliary cirrhosis, extrahepatic biliary obstruction and various systemic infectious diseases. However, Mistilis (13) has shown for the first time that there are similar morphological changes in liver biopsy specimens from patients with chronic ulcerative colitis. Thereafter, the term has been used as a clinical and histological diagnosis for cholangitis related to chronic ulcerative colitis. Therefore, pericholangitis in the case we described may be associated with ulcerative colitis diagnosed by colonoscopy with biopsy.

Regarding autoimmune hepatitis accompanied by ulcerative colitis, Gray et al (14) have reported 8 patients with hepatitis, colitis and lupus manifestations. Willcox and Isselbacher (15) have shown chronic ulcerative colitis as a coexisting disease in 7 of 33 patients with chronic hepatitis which occurred prior to the age of 22 years. Of the 33 patients they described, 22 were female with a mean serum globulin level of $5.8 \pm 2 \mathrm{gm} / \mathrm{dl}$, suggesting that liver dysfunction may be related to an autoimmune mechanism. Olsson and Hultén (16) also have shown that 7 of 25 patients with chronic active hepatitis had coexisting ulcerative colitis. Smooth muscle and antinuclear antibodies were positive in 3 of the 7 patients with ulcerative colitis they described. Judging from these reports, we feel that autoimmune hepatitis may develop ulcerative colitis, although the series reported are extremely small.

In summary, we have presented a patient with pericholangitis associated with ulcerative colitis which occurred presumably through autoimmune mechanisms after a long-term clinical course of autoimmune hepatitis.

\section{References}

1) Mackay IR, Taft LI, Cowling DC. Lupoid hepatitis. Lancet 2: 1323,1956

2) Mistilis SP, Blackburn CRB. Active chronic hepatitis. Am J Med 48: 484, 1970.

3) Soloway RD, Summerskill WHJ, Baggenstoss AH, Schoenfield, LJ. "Lupoid" hepatitis, a nonentity in the spectrum of chronic active liver disease. Gastrocnterology 63: 458, 1972.

4) Wee A, Ludwig J. Pericholangitis in chronic ulcerative colitis; Primary sclerosing cholangitis of the small bile ducts? Ann Intern Med 102: 581, 1985.

5) Chapman RWG, Arborgh BÅM, Rhodes JA, Summerfield JA, Dick R, Scheuer PJ, Sherlock S. Primary sclerosing cholangitis: a rcview of its clinical features, cholangiography, and hepatic histology. Gut 21: 870, 1980.

6) Blackstone MO, Nemchausky BA. Cholangiographic abnormalities in uleerative colitis associated pericholangitis which resemble sclerosing cholangitis. Am J Dig Dis 23: 579, 1978.

7) LaRusso NF, Wiesner RH, Ludwig J, MacCarty RL. Current concepts: Primary sclerosing cholangitis. N Engl J Med 310: $899,1984$.

8) Bodenheimer HC, LaRusso NF, Thayer WR, Charland C, Staples PJ, Ludwig J. Elevated circulating immune complexes in primary sclerosing cholangitis. Hepatology 23: 150, 1983.

9) Zauli D, Schrumpf E, Crespi C, Cassani F, Fausa O, Aadland E. An autoantibody profile in primary sclerosing cholangitis. J Hepatol 5: 14, 1987.

10) Williams SM, Harned RK. Hepatobiliary complications of in flammatory bowel disease. Radiol Clin N Am 25: 175, 1987.

11) Perrett AD, Higgins G, Johnston HH, Massarella GR, Truelove SC, Wright $\mathrm{R}$. The liver in ulcerative colitis. Q J Med 158: 211, 1971.

12) Dordal E, Glagov S, Kirsner JB. Hepatic lesions in chronic inflammatory bowel diseasc. I. Clinical correlations with liver biopsy diagnoses in 103 patients. Gastroenterology 52: 239, 1967.

13) Mistilis SP. Pericholangitis and uicerative colitis. I. Pathology, ctiology and pathogenesis. Ann Intern Med 63: 1, 1965.

14) Gray N, MacKay IR, Taft LI, Weiden S, Wood IJ. Hepatitis, colitis, and lupus manifestations. Am J Dig Dis 3: 481, 1958.

15) Willcox RG, Isselbacher KJ. Chronic liver disease in young people. Clinical features and course in thirty-three patients. Am J Med 30: 185, 1961.

16) Olsson $R$, Hultén L. Concurrence of ulcerative colitis and chronic active hepatitis. Clinical courses and results of colectomy. Scand J Gastrocnterol 10: 331, 1975. 